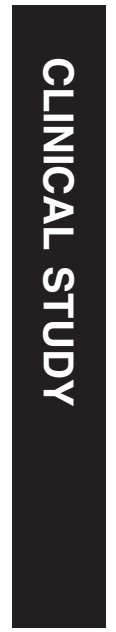

\section{Arrangements for the management of urgent retinal detachments in the United Kingdom and Eire in the year 2000: results of a survey}

Abstract

Purpose To ascertain the current provision of facilities for the management of vitreoretinal (V-R) emergencies, and attitudes of $\mathrm{V}$ $R$ surgeons towards the management of $V-R$ emergencies within the United Kingdom and Eire.

Methods To obtain this information, all 82 consultant members of the Britain and Eire Association of Vitreo-Retinal Surgeons (BEAVRS) were requested to complete a 14part postal questionnaire in the year 2000. Seventy-eight questionnaires were completed and returned. Data from the questionnaires were collated on a Microsoft Access Database and then statistically analysed using SPSS. The Student's $t$-test was used to assess relationships between continuous variables, and the Chi-squared and Fisher's Exact tests were used to compare nonparametric data. Analyses of the first 13 parts of the questionnaire are presented in this report.

Results The majority of V-R surgeons (59\%) practise in teaching hospitals. There are greater numbers of $\mathrm{V}-\mathrm{R}$ surgeons per unit in teaching hospitals as compared to District General Hospitals (DGHs). Ophthalmic theatre and ophthalmic theatre staff availability are theoretically high $\mathbf{( 9 2 . 3 \%}$ and $84.6 \%$ respectively) and evenly distributed between teaching hospitals and DGHs, but in reality, access may be difficult. Most V-R surgeons take part in an on-call rota with general ophthalmology colleagues. This formal commitment may be infrequent. Only a small proportion of V-R surgeons (28.3\%) officially provide a continuous fixed on-call
V-R rota, though in practice, a larger proportion do seem to provide this type of cover informally. Most V-R fellows are located in teaching hospitals $(89.5 \%)$, and are usually on either a formal or informal on-call rota. Only one unit has a formal continuous on-call rota for fellows allowing no more than 72 hours duty per week. The mean time given in response to the question as to the ideal time within which surgery of an acute macula on supero-temporal retinal detachment should be carried out was 29 hours. Most V-R surgeons would not support, in court, a colleague whose patient lost vision through delay in treating a macula on detachment.

Conclusions The findings of this survey have important implications for providing a reliable service and for proper cover for doctors in training.

Eye (2002) 16, 754-760. doi:10.1038/

sj.eye. 6700326

Keywords: retinal detachment; macula; survey; medico-legal

Introduction

Over the last two decades, ophthalmic surgery in the United Kingdom has become increasingly sub-specialised. Vitreo-retinal (VR) surgery was probably the first surgical interest to break away from general ophthalmology, and the one that general ophthalmologists were keenest to give up. Largely, this was due to the increasingly complex surgery required, but may also have been in part because V-R surgery often 
involved emergency surgery out of normal working hours.

Rhegmatogenous retinal detachment remains one of several ophthalmic conditions sometimes requiring urgent surgical intervention. We undertook a survey of the consultant V-R surgical members of BEAVRS to ascertain the current provision of facilities for emergency surgery, the availability of medical and nursing staff, and their on-call arrangements. We tested the attitudes of consultant V-R surgeons to hypothetical situations that could result in medico-legal action.

\section{Materials and methods}

A questionnaire was devised and sent to all consultant members of BEAVRS, an association founded 16 years ago, whose consultant membership includes the majority of specialist V-R surgeons in the British Isles. The questionnaire was designed to obtain information on the management of acute V-R emergencies, attitudes towards management of retinal detachments and facilities available or provided by the National Health Service (NHS) which might influence the provision of emergency care. The responses to 13 questions are analysed in this report.

Eighty-two questionnaires were sent to BEAVRS members on 14 June 2000 and those returned by 14 Sept 2000 numbering $78(95.1 \%)$ were used in this study. The survey was not anonymous, but an undertaking was given that no surgeon would be named in any subsequent communication. Those surgeons (seven) who failed to respond to an initial questionnaire were sent one reminder. Data from the questionnaire were input to a Microsoft Access database and analysed with SPSS and Microsoft Excel. Student's $t$-test was used to assess relationships between continuous variables and the Chi-squared and Fisher's Exact tests were used to compare nonparametric data.

\section{Results}

Seventy-eight BEAVRS consultants responded from 57 ophthalmic units.

\section{Question 1. Describe your ophthalmic unit}

Fifty-nine per cent of the respondents were from teaching hospitals and $47.4 \%$ of the units were teaching hospitals.
Question 2a. At weekends do you have access to an ophthalmic theatre?

Seventy-two (92.3\%) BEAVRS consultants had weekend access to an ophthalmic theatre and 51 (89.5\%) units provided access to ophthalmic theatres on weekends.

\section{Question 2b. At weekends do you have access to ophthalmic theatre staff?}

Sixty-six $(84.6 \%)$ BEAVRS consultants had weekend access to ophthalmic-trained theatre staff and 46 (80.7\%) units provided weekend access to ophthalmictrained theatre staff. There was no statistical difference between teaching hospitals and DGHs in the above two provisions $(P=0.673$ and $P=0.512)$.

Question 3. If no, to either part of question 2, what arrangements for theatre have been made?

For BEAVRS consultants without access to either one or both of the above, there was a variable spectrum of provision. In some instances non-ophthalmic emergency theatres were shared with other specialties, or there was sporadic availability of ophthalmic-trained theatre staff. In others generally trained nurses obtained phoned advice from ophthalmic-trained staff.

\section{Question 4. How many consultant eye surgeons are there on your on call rota? (See Figure 1)}

There were $56(98.2 \%)$ units represented in responses to question number four, showing that the mean number of consultants in on-call rotas was 6.04. The mean number of consultants in teaching hospitals (7.6) was significantly greater than in DGHs (4.7) with a $P$ value of 0.003 .

\section{Question 5. How many consultant vitreo retinal} surgeons are there in your unit? (See Figure 2)

Fifty-seven (100\%) units were represented in responses to question five; the mean number of V-R consultants per unit was 1.61 and again there were significantly more consultants per unit in teaching hospitals (2.1) than in DGHs (1.2) with a $P$ value of 0.001 .

\section{Question 6. Do you have VR Fellows? If yes, how many VR Fellows?}

In the context of this study a fellow is a senior trainee undergoing advanced sub-specialist training in V-R surgery. All units were represented in response to this question; 19 (33.3\%) had V-R fellows and they were 
Consultant on call rota

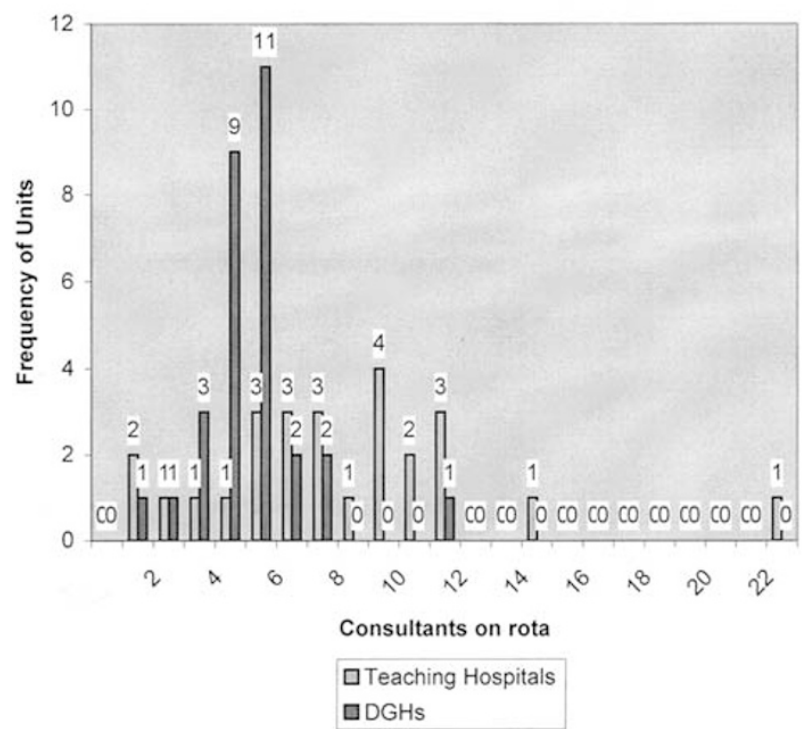

Figure 1 Consultant on-call rota by unit type.

Number of VR Consultants per unit

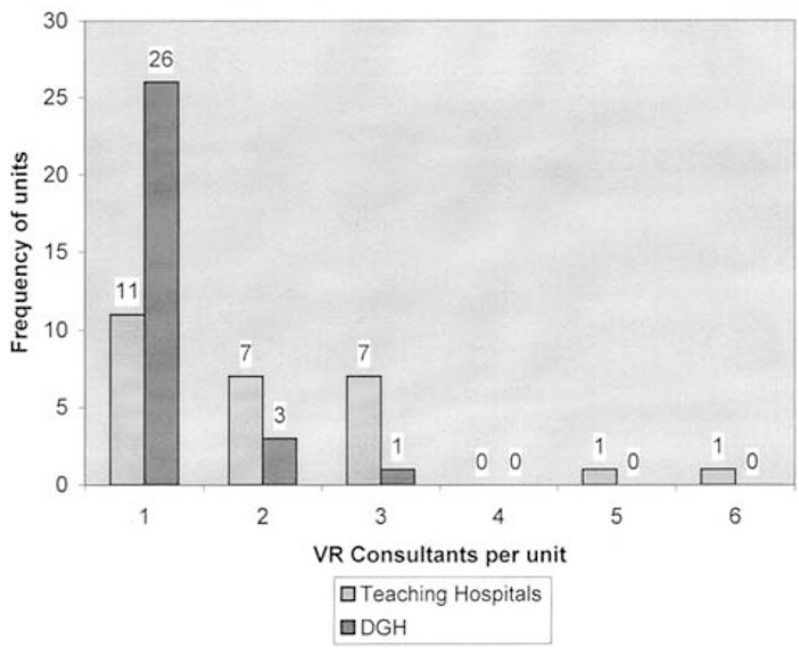

Figure 2 Number of V-R consultants per unit, by unit type.

significantly more likely to be in teaching hospitals than in DGHs $(P=0.001)$ (see Figures 3 and 4$)$. Of the 19 units with V-R fellows, $18(94.7 \%)$ replied to the question as to how many V-R fellows were present. In those units there was an average of 1.5 fellows per unit.

\section{Question 7a. Do you have a VR fellow on-call} rota? If yes please state rota

Eighteen $(94.7 \%)$ units with V-R fellows responded to the first part of question 7 ; three $(16.7 \%)$ of those units
VR Fellows by unit Type

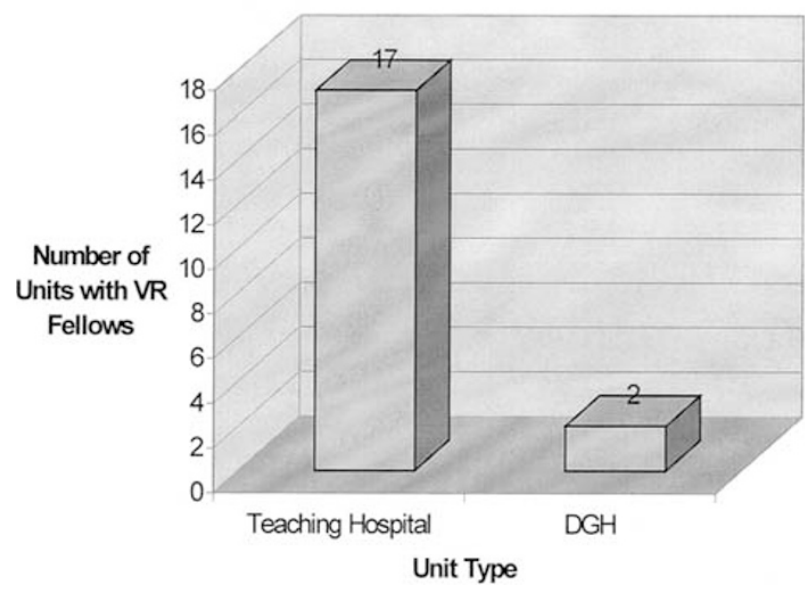

Figure 3 Number of V-R fellows by unit type.

Number of VR Fellows per unit

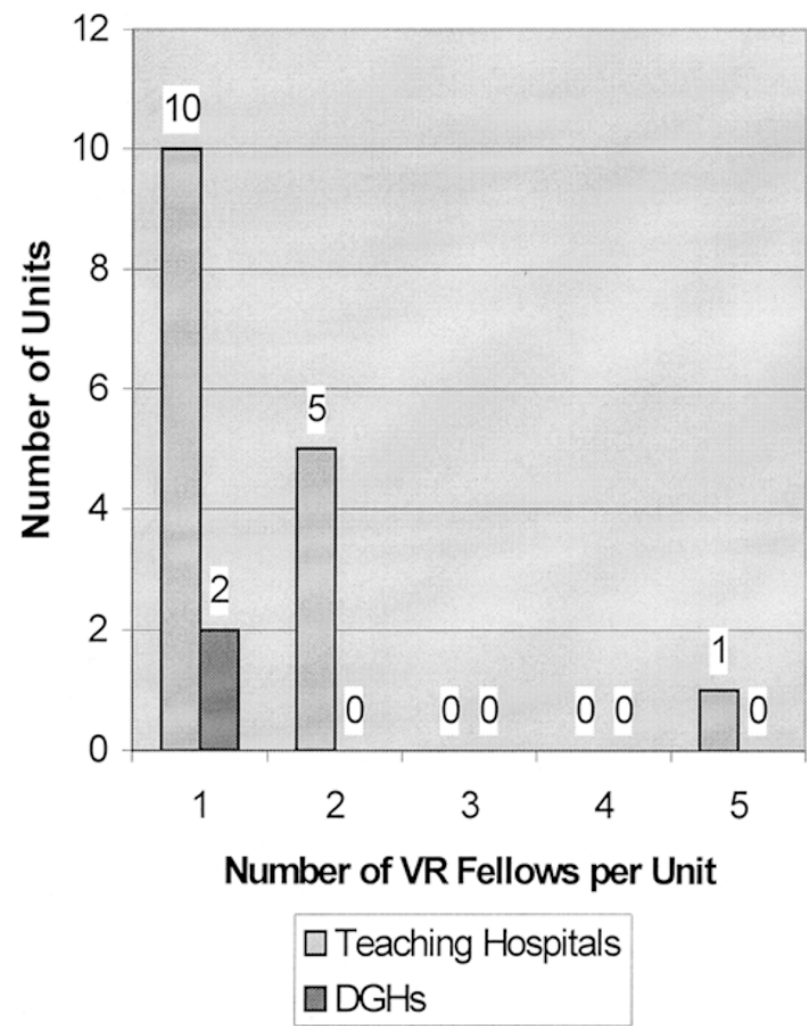

Figure 4 Number of V-R fellows per unit.

stated that they had a formal V-R fellow on-call rota. All of those units were teaching hospitals. The on-call rotas were 1:3, 1:4 and 1:5. 
Question 7b. If there is no formal VR fellow rota is there an informal arrangement so that the VR fellow will do cases when he is available?

All units with fellows responded to the question of whether they had an informal V-R fellow on-call arrangement, and of those $12(57.9 \%)$ gave an affirmative reply.

Question 7c. Is there a consultant VR surgeon on call with the VR fellow?

When asked whether consultant V-R surgeons were on-call with V-R fellows, $16(84.2 \%)$ responded, and of those seven $(43 \%)$ responded in the affirmative and another seven $(43 \%)$ responded that they were sometimes on-call with the V-R fellow.

Question 8. Do general ophthalmologists in your unit do VR surgery?

All units were represented in the answers to question 8 , and of those eight (14\%) units responded that general ophthalmologists did carry out retinal surgery. There was no significant difference between teaching hospitals and DGHs in this respect $(P=0.054)$.

Question 9. Do the consultant VR surgeons in your unit have a rota to provide continuous cover for VR emergencies?

Fifty-three (93\%) units responded to the question, and $15(28.3 \%)$ units reported that V-R surgeons had a rota to provide continuous cover for $\mathrm{V}-\mathrm{R}$ emergencies.

For the next 3 questions, please consider a patient presenting at $5.30 \mathrm{pm}$ on Friday evening with an upper half bullous retinal detachment and $U$ tears. The macula is on:

Question 10. Within what period (in hours) should the patient ideally have VR surgery?

Question 11. If your unit does have an on-call VR surgeon rota (consultant or fellow), when would the patient be operated on?

Question 12. If you do not have an on-call VR surgeon, when would the patient be operated on?

The average time to surgery that most BEAVRS members would ideally like to see for an acute macular on superior retinal detachment was 29 hours with a mode of 24 hours, and a range from 1 to 120 hours. There was no significant difference in timing of surgery whether or not there was an on-call V-R surgeon rota $(P=0.958)$.

Question 13a. A patient with macula on upper bullous retinal detachment and 6/6 vision presents to an eye department (not your own) at $5.30 \mathrm{pm}$ on a Friday. This patient is admitted and undergoes surgery for retinal detachment on Monday pm by which time the vision is reduced to 6/36 because the macula is detached. The operation is technically successful and uncomplicated but the vision fails to improve past 6/24. You are asked to provide a medico-legal opinion as to whether the medical care was of acceptable standard - how would you respond?

With this scenario there was a $96.2 \%$ response rate, and of those, $45(60 \%)$ BEAVRS consultants were of the opinion that medical care was of below acceptable standard, whilst $27(36 \%)$ felt that care had been acceptable. Three (4\%) answered that depending on circumstances either answer could be valid.

Question 13b1. A patient presents to your unit with retinal detachment. The macula is off and has just detached in the last 24 hours. Within what period should the patient undergo surgery?

The response rate was $97.4 \%$ of BEAVRS, and the average time to surgery was 3 days, with a mode of 2 days and a range of 7 hours to 14 days.

Question 13b2. If the macula has been off for one week, within what period should the patient undergo surgery?

For a 1-week macula off retinal detachment the response rate was $93.6 \%$, with an average time to surgery of 2 weeks and a mode of 1 week. There was a significant difference in the timing of surgery for 2week macula off detachments between units where consultants provided continuous vitreo-retinal cover and units which did not $(P=0.015)$. The same was true when units providing weekend ophthalmic theatre staff were compared against those units that did not $(P$ $=0.03$ ).

\section{Discussion}

This study was designed to find out what arrangements are in place for the management of V-R 
emergencies such as acute retinal detachments in the United Kingdom and Eire. Initially, a short questionnaire had been sent to three well-known V-R surgeons in the United Kingdom asking within what timeframe acute retinal detachments with the macula attached were operated on. Various strategies were suggested, and as a result, the study reported here was conceived. The object was to look broadly at the arrangements available to BEAVRS members, and to ask their opinions on how to manage acute retinal detachments.

There is no doubt that anatomical repair of retinal detachments is desirable and that macular on detachments have a better visual outcome. ${ }^{1}$ Burton in $1982^{2}$ and others ${ }^{3}$ have shown a highly significant correlation between postoperative surgical result and visual acuity. He also found that macula off retinal detachments showed a highly significant negative effect on postoperative visual acuity; and that preoperative visual acuity correlated highly significantly with final visual acuity. Burton produced a linear regression model demonstrating an $r^{2}$ value of 0.71 , showing that the duration of macular elevation had a negative effect on final visual acuity. Ross and Kozy in $1998^{4}$ agreed, that in macula off retinal detachments operated on within 7 days, the level of preoperative visual acuity is significantly correlated to postoperative visual acuity. They also made the highly important observation that the length of time that the macula is elevated within the first 7 days has no significant influence on postoperative visual acuity.

The BEAVRS members polled in this study represent a large majority of $\mathrm{V}-\mathrm{R}$ surgeons in the British Isles. More BEAVRS consultants were from teaching hospitals than from DGHs, a finding that is unsurprising, however there were slightly more DGHs represented than teaching hospitals. The finding that there were significantly more V-R surgeons per unit in teaching hospitals than in DGHs $(P=0.001)$, displays the traditional trend towards concentration of this subspecialty in teaching hospitals.

A high proportion of BEAVRS members and ophthalmic units have ophthalmic theatres and ophthalmic theatre staff available on weekends, and there is no statistically significant difference between teaching hospitals and DGHs in this respect $(P=0.673$, $P=0.512$ ). This should mean that emergency surgery could easily be performed, however when theatres and staff are said to be available this does not necessarily mean that surgery can be easily arranged. Often, anaesthetists are shared across several surgical specialties. Theatre staff may take a variable amount of time to arrive and be insufficiently experienced. This is illustrated by the answers to question three, which seek to find out what mechanisms are in place to overcome lack of ophthalmic emergency theatres and/or staff. The answers to this question reveal a very poor level of provision in some units, resulting in a situation that may be difficult to defend medicolegally, and may necessitate referral to another unit with better provision for emergency surgery. Availability per se appears not to be the issue here. Instead, it is the ease of access to, and functionality of, facilities that are crucial.

Most V-R consultants appear to be on a general oncall rota as illustrated by the mean consultants on their rota of 6.04 , being different from the mean number of V-R consultants per unit of 1.61. This certainly has implications for the provision of an emergency V-R service, ie do V-R consultants only provide out of hours cover in their turn as part of the general on-call rota, or do they provide cover at other times when not strictly on call? The latter appears to be true in a large number of cases. There are certainly not enough $\mathrm{V}-\mathrm{R}$ surgeons per unit to generate a reasonable on-call rota for consultants in such a potentially busy subspecialty where, because of lack of adequately trained juniors, active management would usually have to be carried out by consultants themselves.

Only one third of units provide training for V-R fellows, most of which are teaching hospitals $(P=$ 0.001). Presumably the presence of fellows implies that the unit has a caseload large enough to provide the training required, and also enough staff to allow adequate out of hours emergency V-R cover. Most units had only one V-R fellow. In this situation, a continuous out of hours service provided by that junior doctor would be illegal (ie more than 72 hours of work per week). There was only one unit with five fellows allowing a continuous out of hours service from fellows on call.

In the next question three teaching hospitals claimed to have an on-call rota for V-R fellows (1:3, 1:4, 1:5). Again it is not surprising that the units with on-call rotas are teaching hospitals. However, there is a discrepancy in the number of fellows available to provide the rotas stated, unless fellows do not in fact cover some weekends. It is interesting that of the 19 units with fellows, only $16.7 \%$ provide a rota. This displays either the lack of requirement, the low number of suitably trained juniors, or the lack of consultants to provide cover for trainees. $63.2 \%$ of units with V-R fellows however did have an informal arrangement for V-R fellows to carry out emergency cases. Why are there so many units with informal arrangements? The implication is that a large proportion of out of hours V-R work by fellows is on a voluntary basis. With $78.9 \%$ of units with fellows 
providing some form of out of hours service, and only $36.8 \%$ of units where the consultants cover fellows, this suggests that half of the time fellows may not be covered when working out of hours, a potentially dangerous situation. Of course, consultants may actually be providing some form of cover unofficially as fellows would not normally operate without discussing the case with a V-R consultant. However, the lack of formal arrangements indicates a disorganised situation that may put junior doctors and patients at risk.

Only a small proportion of units (14\%) with BEAVRS members also have general ophthalmologists who carry out retinal surgery, though only one of the eight is from a teaching hospital. It is somewhat surprising that in units with V-R surgeons, general ophthalmologists actually still carry out retinal surgery, but as the V-R surgeon cannot reasonably provide continuous cover, this probably should be expected. One would suspect that in units without V-R surgeons a greater proportion of general ophthalmologists carry out basic retinal surgery. This raises the question as to whether non-specialists should carry out V-R surgery, or if retinal surgery should be always referred to a V-R surgeon. Older ophthalmologists may be very experienced in conventional retinal surgery and achieve good enough results to continue, however, newer consultants will probably have little or no practical retinal surgical experience and therefore will refer to a specialist V-R surgeon.

A small number $(28.3 \%)$ of units have consultants providing a continuous V-R rota. This reflects the low number of V-R specialists. Should we be striving to provide continuous cover? If so, either more specialists will be required, or units will need to combine together to provide on-call cover, such that there are enough V-R consultants to provide a reasonable on-call rota.

When BEAVRS members were presented with the first scenario, their mean ideal time within which surgery for an acute macular on retinal detachment should be carried out was approximately one day. The single respondent who suggested surgery within one hour was probably the most idealistic and correct. Facilities and provisions in individual units had no statistical effect on the answers, and should not have done, as the question was about 'ideals'.

There was a degree of discrepancy between answers to question nine and whether question eleven or twelve was answered. It appears that even though VR consultants may not be on a continuous rota, some of them view themselves as effectively being continuously available. Nevertheless, there is no obvious difference in timing of surgery whether or not there is officially a continuous rota $(P=0.958)$. This demonstrates very strongly the unrecognised time that respondents put into out of hours work. It is interesting to note also the wide range of responses, ie a range of 5 days, for the same scenario. The question we need to ask ourselves is whether this situation is really an emergency, requiring emergency surgery, or whether with appropriate alternative management (eg bedrest, posture) surgery can be safely deferred.

How do we view the management of an in-patient who progresses from macular on to macular off over a weekend (second scenario)? Of the $96 \%$ of BEAVRS members who responded, $60 \%$ were of the opinion that management was below an acceptable standard, whilst $4 \%$ felt that depending on circumstances it may or may not be acceptable. A large minority of $36 \%$ were of the opinion that management was acceptable. A large number of responses as to why management was reasonable were given. These included the argument that as long as the patient was rested in bed and suitably positioned the detachment should not progress, and if it did, then all reasonable action had been taken - but is that really so? Previously published work certainly shows that visual results are less good once the macula is detached.

It appears that in some countries it is the norm not to operate on acute macular on retinal detachments over the weekend (personal communication via questionnaire). We wonder if attitudes would be different if theatre access were easier, and if enough suitably trained personnel were made available. We found no single factor that significantly influenced the answer to this question. The nearest to significance was having a continuous $\mathrm{V}-\mathrm{R}$ consultant on-call rota $(P=$ 0.110) which is not that surprising.

The survey showed that a retinal detachment in which the macula had detached 24 hours previously, would, on average, be operated on within 3 days (with a wide range). Though the situation is not an emergency, surgical timing is controversial, with Ross and $\mathrm{Kozy}^{4}$ showing no significant difference in visual outcome from surgery at any time within one week, and Burton's ${ }^{2}$ paper demonstrating that after one week the resultant visual acuity progressively drops.

Bearing in mind the eventual progressive visual deterioration, the survey showed that surgeons repair retinal detachments where the macula has been off for one week, sooner rather than later. In this study the mean time to surgery was 2 weeks. A significant modifier of time to surgery after a one-week macular off retinal detachment was a V-R consultant on-call rota $(P=0.015)$. Surprisingly, the presence of a rota led to a longer time to surgery. This may be due to the acute caseload in those units being so great that less 
urgent cases are pushed back. Spreading the caseload across several units would prevent delay in operating on less acute cases.

This survey shows that, if it is agreed that there are $\mathrm{V}-\mathrm{R}$ surgical conditions requiring emergency surgery, then in almost all areas of the Great Britain and Eire, there is a need for improved organisation to provide adequate facilities and staff so as to provide a reliable, safe service for patients. This may involve collaborative agreements between units. The formulation of best practice guidelines for RD could provide the impetus at local, regional and national level for the necessary changes to be made.

\section{Acknowledgements}

We would like to thank all the members of BEAVRS for their help in promptly replying to our questionnaire, and their individual insights into the problems illustrated by this paper.

\section{References}

1 Norton EWD. Retinal detachment in aphakia. Trans Am Ophthalmol Soc 1963; 61: 770-789.

2 Burton TC. Recovery of visual acuity after retinal detachment involving the macula. Trans Am Ophthalmol Soc 1982; 80: 475-479.

3 Tani P, Robertson DM, Langworthy A. Prognosis for central vision and anatomic reattachment in rhegmatogenous retinal detachment with macula detachment. Am J Ophthalmol 1981; 92: 611-620.

4 Ross WH, Kozy DW. Visual recovery in macula-off rhegmatogenous retinal detachments. Ophthalmology 1998; 105: 2149-2153. 